\title{
Full thickness penetrating keratoplasty with cataract extraction and intraocular lens implantation in leprosy patients
}

\author{
ESSAM EL TOUKHY ${ }^{\mathrm{a}}$ \& NADEEN EL TOUKH Y \\ ${ }^{a}$ Professor of Ophthalmology, Cairo University \& Previous Director, \\ National Eye Centre, Cairo, Egypt \\ ${ }^{\mathrm{b}}$ Undergraduate Student, University of Pennsylvania, Philadelphia, USA
}

\begin{abstract}
Accepted for publication 29 August 2019
Summary

Objective: To evaluate visual acuity outcomes and graft survival after full thickness penetrating keratoplasty (PKP) in leprosy patients.

Design: Case series of triple procedure (PKP, cataract extraction and intraocular implantation) performed on ten eyes of ten leprosy patients who had dense corneal opacity and best correct visual acuity (VA) of $<1 / 60$ preoperatively. Data on preoperative and postoperative VA and ocular complications were extracted from case records of patients. Follow up details were available up to 18 months.

Results: VA improved in eight out of ten patients from $<1 / 60$ preoperatively to a range of $5 / 60$ to $6 / 24$ postoperatively at 6 months and the VA was stable at 12 months. Two patients had graft failure with no improvement in VA. Four patients had mild to moderate graft rejections that responded satisfactorily to medical treatment. Epithelial erosion was observed in all patients and was the only complication seen in four patients. None of the epithelial erosions progressed to deep ulcers or epithelial reactions.

Conclusion: In the majority of eyes, visual acuity improved after corneal transplantation. PKP should be offered to blind leprosy patients who have the potential to recover vision, after carefully individualizing the risks and benefits.
\end{abstract}

Keywords: Corneal grafts, leprosy, blindness, cataract in leprosy, corneal opacity

\section{Introduction}

Leprosy is a chronic infectious disease caused by Mycobacterium leprae that primarily affects peripheral nerves and skin. The eyes are frequently affected leading to loss of vision, ${ }^{1,2}$ with corneal opacities and ulcers being the leading causes of blindness in leprosy. ${ }^{3,4}$ A number of co-morbidities predispose the eyes of leprosy patients to corneal ulcers and opacities including lagophthalmos, reduced or absent corneal sensation and altered tear functions. ${ }^{5-10}$ Ocular morbidity and blindness are devastating complications in leprosy patients, especially those with Grade 1 and Grade 2 disabilities for whom good vision is critical in protecting their

Correspondence to: Essam El Toukhy, MD, Cairo University, Dokki, Cairo, Egypt 14-A El Sobki Street \#23, Dokki, Cairo, Egypt. 12311 (e-mail: eeltoukhy@yahoo.com) 
anesthetic limbs. ${ }^{11}$ Most dense corneal opacities that cause blindness and preclude good visual acuity (VA) can only be managed by full thickness penetrating keratoplasty (PKP). In leprosy patients, however, a literature search shows the scarcity of this surgical procedure with just one case report of a corneal transplantation being done half a century ago. ${ }^{12}$ The reluctance to perform this surgery in leprosy has been attributed to the increased occurrence of well recognized risk factors to poor visual outcomes and graft rejection such as exposure keratitis due to lagophthalmos, reduced corneal sensation and abnormal tear function tests. There are also other reasons, but outcomes after corneal transplantation in the non-leprosy population have been very successful in the past few decades. This has largely been due to improvements in the surgical techniques, better instrumentation and better postoperative care. ${ }^{13,14,17-19}$ It appears that even with these advancements this procedure has not been applied to leprosy patients. For leprosy patients with corneal blindness with potential to improve vision after PKP, it could be a life changing procedure. We present in this report, the one year outcomes of a series of blind leprosy patients who underwent PKP for dense corneal opacities.

\section{Materials and methods}

From 2016 to 2018, leprosy patients from Abu Zabal in Egypt were selected to undergo corneal transplantation and were operated on at the leprosy clinic, Kalaa in Cairo, in collaboration with leprosy control department, Ministry of Health, Egypt. Inclusion criteria for patients were: completion of a full course of multi-drug therapy (MDT) at least two years before surgery; no reactions for at least 1 year before surgery; blind with visual acuity $<3 / 60$ in both eyes; lagophthalmos, if present, must have been surgically treated at least 6 months before the corneal grafting; and dense corneal opacities in the study eye with a visual acuity of less than 1/60. Study eyes must have a potential for visual acuity recovery evidenced by a comprehensive ophthalmic examination including intraocular tension, electro-retinogram and visually evoked potential.

\section{PERI-SURGICAL PROCEDURES}

After the risks of the surgical procedure were explained to the patient and informed consent was obtained, patients were placed on topical dexamethasone eye drops four times daily, two weeks before surgery. To reduce corneal neovascularization, an injection of bevacizumab (Avastin ${ }^{\circledR}$ ) was given in the area of highest limbal vascularization one week before surgery. Full thickness corneal grafts obtained from USA were used in the transplant.

A $7 \mathrm{~mm}$ penetrating corneal graft was done under local anesthesia. Concomitant extracapsular cataract extraction with intra-ocular lens implantation was performed in all cases. The graft was sutured with 10/0 nylon sutures in an interrupted or continuous manner. Adhesions between the iris and cornea or the lens, if present, were released. An antibiotic steroid combination was injected in the lower fornix at the completion of surgery. An eye patch was used postoperatively until epithelial healing was complete. Antibiotic eye drops were used 5 times daily for 1 week and then the dose was tapered over a month. Steroids eye drops were used every two hours for the first week and then 5 times daily for one month and were adjusted depending on the inflammatory response.

An antibiotic steroid ointment was used at night in all cases for a month and then replaced by tobramycin eye ointment for life. Postoperatively, lubricants were used several times during the day to prevent dryness. No systemic steroids were given. Patients were examined on days $1,3,5$ and 7 postoperatively, then weekly for the first 2 months and then monthly for the first 
Table 1. Patient and leprosy characteristics

\begin{tabular}{lccccc}
\hline Patient & Sex & Age (years) & Type of leprosy* & $\begin{array}{c}\text { Duration of } \\
\text { leprosy (years) }\end{array}$ & $\begin{array}{c}\text { Prior Lagophthalmos } \\
\text { surgery** }\end{array}$ \\
\hline 1 & F & 68 & MB & 30 & Yes \\
2 & F & 51 & MB & 15 & No \\
3 & M & 32 & MB & 12 & Yes \\
4 & F & 49 & PB & 28 & Yes \\
5 & M & 50 & MB & 20 & No \\
6 & F & 57 & PB & 32 & No \\
7 & F & 56 & MB & 25 & Yes \\
8 & M & 42 & MB & 10 & No \\
9 & F & 60 & MB & 35 & No \\
10 & M & 55 & MB & 30 & \\
\hline
\end{tabular}

${ }^{*}$ WHO clinical classification. MB $=$ Multi-bacillary PB $=$ Paucibacillary. ${ }^{* *}$ Lagophthalmos, if present with $<2 \mathrm{~mm}$ of palpebral width, was surgically treated at least 6 months prior to corneal grafting. None of the patients had a lagophthalmos with exposure of $>2 \mathrm{~mm}$ at the time of corneal grafting. No lagophthalmos surgery was performed at the time of corneal grafting.

6 months. Patients were instructed to return immediately in case of any redness, pain and/or decrease in vision. In two cases the corneal sutures were removed only at 10 and 12 months post-operatively. Topical antibiotic steroid eye drops were used for two weeks in these two cases to avoid any risk of infection or inflammation.

\section{Results}

Characteristics of patients and their ocular conditions are given in Table 1. Among 10 patients, 6 were female. Ages ranged from 32 to 68 years (mean 52 years). The mean duration of the disease was 24 years (range 10 to 35 years). Preoperative corneal sensation was absent in all cases because of severe corneal opacity. Except for 2 cases, all were multibacillary leprosy patients. Half of the patients had had prior lagophthalmos surgery.

Table 2 gives the visual acuity at baseline, 2 month, 6 months and 12 months and the complications post-operatively. All patients had corneal opacity in the visual axis and the opacities were dense ( 6 with leucomatous opacities and the rest with macular opacities). Follow-up ranged from 6 months to 18 months but VA was not available for 3 patients at 12 months. All patients had a VA of worse than 1/60 with best corrected vision preoperatively. At the 6 months visit, 8 patients had a clear graft in the visual axis. In two patients the VA never recovered: one of them had primary graft failure, the graft being edematous from the first postoperative day, never regaining clarity and accompanied by a severe inflammatory reaction in the anterior chamber that did not improve in spite of increasing the frequency of steroids eye drops. The other case developed signs of graft rejection after 6 weeks post operatively that progressed to severe corneal edema with bullae and endothelial rejection despite intense steroid treatment. In the remaining eyes at 6 months $(80 \%)$ there was marked improvement in VA that ranged from $5 / 60$ to $6 / 18$. The VA in these eyes was observed to be stable at 12 months but data on VA for three eyes were missing at 12 months. Epithelial erosions occurred in almost all cases in the early post-operative weeks and were the only complications observed in 4 eyes but none of them progressed to severe ulceration or complete epithelial rejection. The rest of the eyes had graft rejections varying in intensity from mild to severe. Mild to moderate graft rejections (inflammatory cells in the anterior chamber and on the endothelium) responded favorably to intense topical steroid therapy and were maintained on a single daily dose of steroid eye drops. 
Table 2. Preoperative and postoperative visual acuity (VA) and ocular characteristics

\begin{tabular}{lcclcccc}
\hline Patient & $\begin{array}{c}\text { Pre-op } \\
\text { VA }^{*}\end{array}$ & $\begin{array}{c}\text { Type of } \\
\text { corneal } \\
\text { opacity }\end{array}$ & $\begin{array}{c}\text { Follow- } \\
\text { up time } \\
\text { (months) }\end{array}$ & $\begin{array}{c}\text { Post-op } \\
\text { VA (2 } \\
\text { months) }\end{array}$ & $\begin{array}{c}\text { Post-op } \\
\text { VA (6 } \\
\text { months) }\end{array}$ & $\begin{array}{c}\text { Post-op VA } \\
(1 \text { year) }\end{array}$ & Complications \\
\hline 1 & $50 \mathrm{~cm}$ & Leucoma & 12 & $6 / 60$ & $6 / 60$ & $6 / 60$ & Epithelial erosions \\
2 & $\mathrm{HM}$ & Leucoma & 14 & $6 / 24$ & $6 / 24$ & $6 / 24$ & Graft rejection \\
3 & $1 / 60$ & Macular & 12 & $5 / 60$ & $6 / 60$ & $6 / 60$ & Epithelial erosions \\
4 & $1 / 60$ & Macular & 18 & $6 / 18$ & $6 / 24$ & $6 / 24$ & Epithelial erosions \\
5 & $70 \mathrm{~cm}$ & Macular & 6 & $\mathrm{HM}$ & $\mathrm{HM}$ & Missing & Primary graft failure \\
6 & $\mathrm{HM}$ & Leucoma & 12 & $\mathrm{HM}$ & $\mathrm{HM}$ & $\mathrm{HM}$ & Graft rejection \\
7 & $30 \mathrm{~cm}$ & Leucoma & 12 & $6 / 60$ & $6 / 60$ & $6 / 60$ & Graft rejection \\
8 & $60 \mathrm{~cm}$ & Leucoma & 18 & $4 / 60$ & $5 / 60$ & $5 / 60$ & Graft rejection \\
9 & $1 / 60$ & Macular & 7 & $6 / 60$ & $5 / 60$ & Missing & Graft rejection \\
10 & $\mathrm{HM}$ & Leucoma & 9 & $6 / 60$ & $6 / 60$ & Missing & Epithelial erosions \\
\hline
\end{tabular}

${ }^{*} \mathrm{HM}=$ Hand Movements.

Examples of an eye with a successful graft in which the VA changed from hand movements before surgery to $6 / 24$ during the post-operative period and another eye that did not improve from a preoperative VA of hand movements is shown in the Figure.

\section{Discussion}

In a series of 10 cases we have shown that penetrating keratoplasty is moderately successful in improving visual acuity within a one-year follow-up time period. Severe graft rejection was observed only in 2 cases.

The solitary report of a case of penetrating keratoplasty done nearly half a century ago for corneal opacity with associated blindness in a leprosy patient is a testament to the reluctance of ophthalmologists to perform these surgeries in leprosy. There are several reasons for the paucity of this surgical procedure in ocular leprosy. There is a fundamental belief among physicians and ophthalmologists working in the field of leprosy that most eyes that warrant this major ophthalmic surgery are likely to have multiple risk factors that increase the likelihood of graft rejection. These include one or more of the following: corneal opacities that extend to the limbus, increased corneal vascularization, dry eye, decreased corneal sensation, lagophthalmos, other lid deformities, and chronic iridocyclitis with its sequelae. It is also likely that in places around the globe where there are pockets of endemic leprosy and in places where corneal opacities are a major concern among bacteriologically cured leprosy patients, there are no specialized eye care facilities or experienced corneal specialist surgeons to perform this major eye surgery. For a leprosy patient who is blind, recovery of vision, however small, is of tremendous benefit leading to a much better status of independence and safety.

It has been shown that cataract surgery with intraocular lens implantation can be performed safely in leprosy patients with good results. ${ }^{13,14}$ Out of the 10 cases in our study, only 7 had significant cataract requiring extraction. We performed cataract surgery with intraocular lens implantation in all 10 cases in order to avoid any future surgery that could put the corneal graft at risk - anticipating the inevitability of cataract development at a later period. Cataracts are common among leprosy patients and could be age related, secondary to iridocyclitis, secondary to steroid therapy or simply as a delayed consequence of the penetrating keratoplasty. The likelihood of cataract formation and cataract extraction subsequent to PKP increases greatly after 50 years of age, regardless of the diagnosis leading to the need for keratoplasty. ${ }^{17}$ 


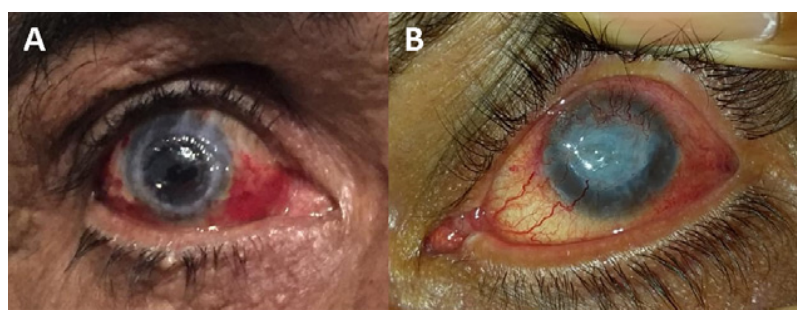

Figure 1. A shows the colour image of an eye with a preoperative visual acuity of $<1 / 60$ which improved to $6 / 24$ postoperatively after penetrating keratoplasty was performed for dense corneal opacity. The visual acuity remained stable at 12 months. The clear corneal button with a slightly horizontally stretched pupil can be seen. B shows an eye that had a dense corneal opacity preoperatively and had penetrating keratoplasty. Postoperatively despite intensive treatment, the eye had a severe graft rejection ending in complete opacification of the cornea and no improvement in visual acuity.

Therefore in leprosy patients undergoing PKP, a triple procedure that combines PKP with phacoemulsification and intraocular lens implantation avoids an almost definite future intraocular surgery that is a potential risk for the wellbeing of a successful graft. ${ }^{18,19}$

In non-leprosy patients, the success rate of corneal grafting can be as high as $90 \%$ and there is restoration of a clear visual axis and improvement in visual acuity. ${ }^{20-22}$ Most of the good VA outcomes were observed in patients who undergo PKP for keratoconus and corneal dystrophies. Graft rejections were higher in cases where the etiology of the corneal opacity was different. ${ }^{23-25}$ It is important to recognize that a substantial number of PKP will end up with a worse prognosis. This is particularly true when the corneal opacity is large, extending to the limbus and where the vascularization is deep and profuse. ${ }^{26-28}$ Preoperative patient selection and the compliance of the patient in combating complications is critical in obtaining good and stable vision postoperatively. The corneal buttons we used for PKP were supplied from the United States. It has been shown that our outcomes would have been no different if we had used corneas donated from the local population. ${ }^{29}$

All the patients in our case series were blind according to the World Health Organization classification. Even the possibility of a small increment in visual acuity would have outweighed the risks for the surgery. But patient selection should still be judicious and the preparation before surgery meticulous. Patients should have the potential to recover visual acuity and must have at least perception of light. They must be well informed so that they correctly understand the risks and the long term care that would be required after the surgery. Patients with deformities may require even more specialized care and frequent oversight. We believe that, despite a higher risk of failure than that which is currently present in the non-leprosy population, PKP should be considered in blind leprosy patients with corneal opacities. In the current scenario of better equipped eye hospitals, experienced ophthalmology surgeons, unique instrumentation, a more knowledgeable public, decreasing stigma towards the disease and excellent drugs to combat inflammation and graft rejection, our series of patients with PKP surgery should inform the leprosy field of the advantages and risks of PKP in combating blindness, especially in an older generation of pre-MDT blind patients with deformities. This procedure could also be offered to a considerable number of cured leprosy patients with cataract and coexisting central leucomatous corneal opacity in whom only an extracapsular cataract extraction and intraocular lens implantation was performed with a pupillary sphincterotomy in lieu of a PKP. ${ }^{30}$ 


\section{Guarantor}

\section{Dr. Essam El Toukhy}

\section{Author contribution}

Both Dr Essam El Toukhy and Nadeen El Toukhy have made a substantial contribution to the conception or design of the work being reported, including the acquisition, analysis, and/ or the interpretation of data for the work. They have participated in the drafting the work and revising it critically for important intellectual content; they have approved the final version submitted, and finally published.

\section{Conflicts of interests}

\section{Both authors report no conflict of interest.}

\section{References}

1 Mondal KK, Biswas S. Review of ocular leprosy. J. Indian Med Assoc, 2006; 104: 401-403.

2 Daniel E, Flytche TJ, Sundar Rao PS, Kempen JH, Diener-West M, Courtright P.. Incidence of ocular morbidity among multibacillary leprosy patents during a 2 year course of multidrug therapy. Br. J Ophthalmol, 2006; 90: 528-529.

3 John D, Daniel E.. Infectious keratitis in leprosy. Br J Ophthalmol, 1999; 83: 173-176.

4 Rohatgi J, Dhaliwal U, Singal A. Factors associated with sight threatening lesions of leprosy in patients on multidrug therapy. J Indian Med Assoc, 2004; 102: 297-298.

5 Mypet C, Hogeweg M. Lid surgery in patients affected with leprosy in North-Eastern Nigeria: Are their needs being met?. Trop Doct, 2006; 36: 11-13.

6 Hogeweg M, Keunen JE. Prevention of blindness in Leprosy and the role of the vision 20/20 project. Eye, 2005; 19: 1099-1105.

7 Lamba PA, Rohatgi J, Bose S. Factors influencing corneal involvement in Leprosy. Int J Lepr Other Mycobact Dis, 1987; 55: 667-671.

8 Koshy S, Daniel E, Kurian N, Yovan P. Pathogenesis of dry eyes in leprosy and tear functions. Int J Lepr Other Mycobact Dis, 2001; 69: 215-218.

9 Courtright P, Daniel E, SundarRao PS, Ravanes J, Mengistu F, Belachew M, Celloria RV, Ffytche T. Eye disease in multibacillary leprosy patients at the time of their leprosy diagnosis: Findings from the Longitudinal Study of Ocular Leprosy (LOSOL) in India, the Philippines and Ethiopia. Lepr Rev, 2002; 73: 225-238.

10 Courtright P, Lewallen S, Li HY, Hu LF, Yang JW. Lagophthalmos in a multibacillary population under multidrug therapy in the People's Republic of China. Lepr Rev, 1995; 66: 214-219.

11 Malik ANJ, Morris RW, Ffytche TJ. The prevalence of ocular complications in leprosy patients seen in the United Kingdom over a period of 21 years. Eye, 2011; 25: 740-745.

12 Jones RF. Keratoplasty in the keratitis of leprosy. Br J Ophthal, 1963; 47: 248-249.

13 Anand S, Neethiodiss P, Xavier JW. Intra and post operative complications and visual outcomes following cataract surgery in leprosy patients. Lepr Rev, 2009; 80: 177-186.

14 Batistella GG, Maakaroun M, de Castro AV. Extracapsular cataract extraction and intraocular lens implantation in leprosy patients: visual outcome and complications. Indian J Lepr, 1998; 70: 5-10.

15 Omar N, Bou Chacra CT, Tabbara KF. Outcome of corneal transplantation in a private institution in Saudi Arabia. Clin Ophthalmol, 2013; 7: 1311-1318.

16 Hussain Farooqui J, Sharifi E, Gomaa A. Corneal surgery in the flying eye hospital: Characteristics and visual outcome. Can J Ophthalmol, 2017; 52: 161-165.

17 Martin TP, Reed JW, Legault C, Oberfeld SM, Jacoby BG, Yu DD, Dickens A, Johnson HP. Cataract formation and cataract extraction after penetrating keratoplasty. Ophthalmology, 1994; 101: 113-119.

18 Meyer RF, Musch DC. Assessment of success and complications of triple procedure surgery. Am J Ophthalmol, 1987; 104: 233-240.

19 Pedersen OO. Combined corneal transplantation, extracapsular cataract extraction, and artificial lens implantation (triple procedure). Acta Ophthalmol Suppl, 1987; 182: 83-86.

20 Chen MC, Kunselman AR, Stetter CM, Hannush SB, Roberts BW. Corneal transplantation at Tenwek Hospital, Kenya, East Africa: Analysis of outcomes and associated patient socioeconomic characteristics. PLoS One, 2017; 12: $\mathrm{e} 0187026$. 
21 Yorston D, Wood M, Foster A. Penetrating keratoplasty in Africa: graft survival and visual outcome. $B r J$ Ophthalmol, 1996; 80: 890-894.

22 Dandona L, Naduvilath TJ, Janarthanan M, Ragu K, Rao GN. Survival analysis and visual outcome in a large series of corneal transplants in India. Br J Ophthalmol, 1997; 81: 726-731.

23 Price MO, Thompson RW, Price FW. Risk factors for various causes of failure in initial corneal grafts. Arch Ophthalmol, 2003; 121: 1087-1092.

24 Ayalew M, Tilahun Y, Holsclaw D, Indaram M, Stoller NE, Keenan JD, Rose-Nussbaumer J. Penetrating keratoplasty at a tertiary referral center in ethiopia: Indications and outcomes. Cornea, 2017; 36: 665-668.

25 Tilahun Y, Shimelash D. The outcome of corneal transplantation versus indications in a tertiary eye care center in Ethiopia. Ethiop Med J, 2010; 48: 35-39.

26 Mohan M, Panda A, Kumar TS. Results of penetrating keratoplasty in vascularized corneas. Ann Ophthalmol, 1990; 22: 235-238.

27 Di Zazzo A, Kheirkhah A, Abud T, Goyal S, Dana R. Management of high-risk corneal transplantation. Surv Ophthalmol, 2017; 62: 816-827.

28 Maguire MG1, Stark WJ, Gottsch JD, Stulting RD, Sugar A. Fink NESA. Risk factors for corneal graft failure and rejection in the collaborative corneal transplantation studies. Ophthalmology, 1994; 9: 1536-1547.

29 Ababneh $\mathrm{OH}, \mathrm{AlOmari}$ AF. Outcomes of penetrating keratoplasty with imported corneas compared with local corneas. Cornea, 2016; 35: 1211-1215.

30 Sinha R, Sharma N, Vajpayee RB. Visual outcome of cataract surgery with pupillary sphincterotomy in eyes with coexisting corneal opacity. BMC Med, 2004; 2: 10 . 